\title{
Managing the Challenges of Becoming an Open Innovation Company: Experiences from Living Labs
}

\section{Mika Westerlund and Seppo Leminen}

\author{
(6 The future cannot be predicted, but futures can be") \\ invented. It was man's ability to invent which has \\ made human society what it is.
}

Dennis Gabor

Physicist, Inventor, and Nobel Laureate (1900-1979)

\begin{abstract}
High-technology firms have paved the way for user-driven innovations, but now even traditional industries are becoming increasingly open. This shift is a great challenge for companies with instituted practices, policies, and customer relationships. In this article, we identify four distinct steps in becoming an open innovation company based on our recent research into firms' experiences with living lab experiments in the information and communication technology (ICT) sector. We describe these phases and illustrate the divergent roles that users play in each one. We conclude with a discussion on the differences between the management challenges of conventional development projects versus the open innovation model. For all firms that wish to become open innovators, we recommend that their managers promote an open organizational mindset and apply groupware that supports increased openness, because traditional project management tools are insufficient for open innovation.
\end{abstract}

\section{Introduction}

Today's organizations need a constant flow of novel ideas while competing through emergent technologies. A growing number of companies pay close attention to users as a source of valuable feedback and relevant use experiences. Companies in all industries agree that integrating users in the innovation process - to learn from and with them - is crucial. Moreover, one of the most important recent trends is the progressive inclusion of users in firms' processes where value is co-created, as described in the November 2009, December 2009, and March 2011 issues of this publication (http://timreview.ca/issue-archive). Co-creation with users helps firms better address their customers' latent needs. It reduces market risk in the launch of new products and services, and it improves return on investment and time to market. Firms involve users in the coproduction of brands, experiences, design, marketing strategies, and products or services.

The increasingly fashionable concept of "open innovation" drives user involvement. It provides an interesting alternative to conventional in-house development and includes various possibilities, such as open sourcing and crowdsourcing. Open source is a widespread means of innovation in the software industry, where open source communities act as innovation intermediaries and peer-to-peer production resources. Examples of well-known open source software projects include the mainstays of the LAMP stack (http://wikipedia.org/wiki/LAMP_(software_bundle)). As for crowdsourcing, Google has been crowdsourcing mapping data, content, and ideas (http://project10tothe100.com) for some time now, and InnoCentive (http://wikipedia .org/wiki/InnoCentive) crowdsources $\mathrm{R} \& \mathrm{D}$ for biomedical and pharmaceutical companies by providing connection and relationship management services between solution seekers and solvers.

One particularly interesting form of open innovation is the living labs approach (http://en.wikipedia.org/wiki/ Living_lab), where technology is developed and tested in a physical or virtual real-life context, and users are important informants and co-creators in the tests (Kusiak, 2007; http://tinyurl.com/5vggb7h). The living labs approach is also attractive for traditional industries, because it extends the conventional innovation processes rather than reinvents them. Companies, on average, have little experience in open innovation, and 


\section{Managing the Challenges of Becoming an Open Innovation Company}

Mika Westerlund and Seppo Leminen

transforming from an in-house innovator into an open innovation company is especially difficult for firms in traditional industries. Existing academic studies (e.g., Almirall and Casadesus-Masanell, 2010; http://tinyurl .com/6je6gph) can offer only limited insight; they predominantly consider firm's innovation development options as either closed or open without indicating what is needed for a firm to become an open innovator.

In this article, we examine the steps and managerial challenges firms face on their way to becoming open innovation companies. First, we look at customer involvement in development work and discuss why some firms choose living labs as their preferred way to initiate it. Second, we describe four different steps of co-creation with customers and users. We use data from our recent research to explain how the role of users and the depth of their integration within firm's innovation processes vary between the four steps. Third, we discuss the organizational challenges of managing co-creation, most of which relate to coping with change from a psychological perspective, because established corporate culture and practices often hinder this type of change. We argue that the managerial tools required in open innovation differ from those used in conventional, projectbased innovation development.

\section{Customer Involvement in Development Work}

Many companies no longer attempt to grasp the details of customer needs and use experiences. They reassign the design aspect of product development to external sources of ideas, such as their customers, who can help with innovation work and create value (Edvarsson et al., 2010; http://tinyurl.com/3exkqua). Seeking to understand user needs is expensive and labour intensive, but customer insight speeds up the development processes of products and services and lowers the cost. Zaltmann (2003; http://tinyurl.com/4xmrtba) argues that firms increasingly recognize the need for integrating users as co-developers in $R \& D$ activities, because at least $80 \%$ of new products and services fail once they are launched into the market. With co-development, the result is more innovative and better fits with market needs.

The most common means of integrating users into development work involves collecting feedback on a company's products and services. However, users are now so intimately involved in the development processes that they have become co-creators of value and the in- novation is user-driven. To co-create value, the firm, its customers, and its partners must reconcile their objectives, define the role and effort required from each party, and agree on an equitable division of the returns (Chesbrough, 2003; http://tinyurl.com/455m3q6). Shifting the focus from ownership to openness requires a total reconsideration of the processes that underlie value creation and capture (Chesbrough and Appleyard, 2007; http://tinyurl.com/3ne6xts).

Customer involvement in innovation development also has challenges. Experiments show that ideas from users are often more original and valuable, but ideas from inhouse developers are more realizable (Edvarsson et al., 2010; http://tinyurl.com/3exkqua). Therefore, managers need to consider the type and organization of $R \& D$ to be performed, including a choice about the exposure of the innovation work to knowledge from outside the firm. Open innovation calls for a specific organizational mindset, which requires the creation and learning of a new operational culture, including open organization, processes, and products and services. Openness is difficult for firms where conventional thinking is the norm, because it means the firm must consider the inputs of others and cannot exert exclusive rights over the resultant innovation.

\section{The Living Lab as a Form of Open Innovation}

A firm can become an open innovator in different ways. Living labs provide an option for firms in industries, where the cognitive distinction between closed and open innovation is particularly strong. Living labs are co-creation ecosystems for human-centric research and innovation. We share the view of Ballon and colleagues (2005; http://tinyurl.com/5wwollx), who define living labs as experimentation environments; they are physical regions or virtual realities where stakeholders form public-private-people partnerships (4Ps) of firms, public agencies, universities, institutes, and users all collaborating for creation, prototyping, validating, and testing of new technologies, services, products and systems in real-life contexts. Living labs are different from test beds for controlled testing of a technology in a laboratory environment and field trials for testing in a limited, but still real-life, environment.

Stewart (2007; http://tinyurl.com/6cx2pfb) makes a distinction between diverse types of living labs. They include: i) narrow but sizable communities of expert users; ii) whole bounded populations; iii) living labs for technical service development; and iv) living labs for 


\section{Managing the Challenges of Becoming an Open Innovation Company}

\section{Mika Westerlund and Seppo Leminen}

non-technical research using a service platform. All these types have something in common: they employ an array of participants with different rationale for joining the innovation development. Participants must reconcile their objectives and define both the role and effort required from each party and an equitable division of the returns to co-create value. Many living labs also join regional or global networks of living labs, such as the geographically distributed European Network of Living Labs (http://www.openlivinglabs.eu).

A living lab provides a concrete setting, unlike the other forms of open and collaborative innovation (Schaffers et al., 2007; http://tinyurl.com/6x8y6ku). Its main activities are:

1. Co-creation: co-design by users and producers; utilizers and enablers are also involved.

2. Exploration: discovering emerging usages, behaviours, and market opportunities.

3. Experimentation: implementing live scenarios within communities of users.

4. Evaluation: assessment of concepts, products, and services according to socio-ergonomic, socio-cognitive, and socio-economic criteria.

Living labs are platforms that bring together all the relevant parties for innovation co-creation. They open up the possibility to generate a wide and extensive spectrum of product and service portfolios (De Ryuter et al., 2007; http://tinyurl.com/3ugxd54) and connect producers and users with utilizers and enablers. The utilizer is a non-producer firm that seeks efficiency gains, supplements to resource bottlenecks, and knowledge from the living lab. It may boost its innovation process through the living lab network or even outsource its innovation capacity and knowledge to boost the living lab network. Enablers are companies or organizations that provide supportive technology, virtual or physical space, and other necessary resources to the use of participants.

\section{Data Collection and Analysis}

Between 2007 and 2010, we conducted 27 semi-structured interviews with senior managers of ICT companies. The data includes companies of all sizes from startups to large multinationals. We chose the ICT industry because open innovation practices are most advanced in high-technology industries (Chiaroni et al.,
2011; http://tinyurl.com/3h4pdav). Therefore, we expected to find many firms that integrate users in their $\mathrm{R} \& \mathrm{D}$ processes or provide such services to other firms. Most of the firms we studied followed closed, producerled development practices, while some were more open and user driven. Because all our case companies employ or intend to use living labs to boost their business, we expected that our interviewees could provide useful information on the past or current challenges of opening up a firm's innovation development. To further understand living labs as a form of open innovation, and the challenges of operating with multiple parties who have different motives, we conducted an additional 40 interviews with the staff of living labs in Finland, Sweden, and Spain.

We analyzed the data in a way similar to Lazzarotti and Manzini (2009; http://tinyurl.com/3zk9zbo), who established a framework to describe four basic ways to collaborate. Although the two dimensions along which we analyzed our data - the degree of openness and the type of co-creation - are different from their study, we also ended up with four different steps of collaboration. The following sections describe these four steps.

\section{Four Steps to Becoming an Open Innovator}

According to our analysis, when a conventional inhouse developer decides to become an open innovation company, they will likely encounter four steps of development: i) producer-driven; ii) user-centric closed; iii) user-centric open; and iv) user-driven, as illustrated in Figure 1. These steps represent increasing degrees of user involvement. Firms are not required to progress through these steps sequentially, although that is the usual pattern. Furthermore, a firm can start or stop at any step. Previous research shows that it may take a long time for a firm to become an open innovator and this change may bring about many challenges (Chiaroni et al., 2011; http://tinyurl.com/3h4pdav). Managers need to establish a new organizational culture and mindset to support opening up their innovation processes.

Step 1: Producer-driven. In the first step, development work is led by the producer and is closed. This step is characterized by technology push, since the innovation originates from the producer's ideas and patents. The firm's policy to maintain knowledge and intellectual property rights within the company guides the development work. The staff has little communication or interaction with users; it considers them merely as buyers 


\section{Managing the Challenges of Becoming an Open Innovation Company}

\section{Mika Westerlund and Seppo Leminen}

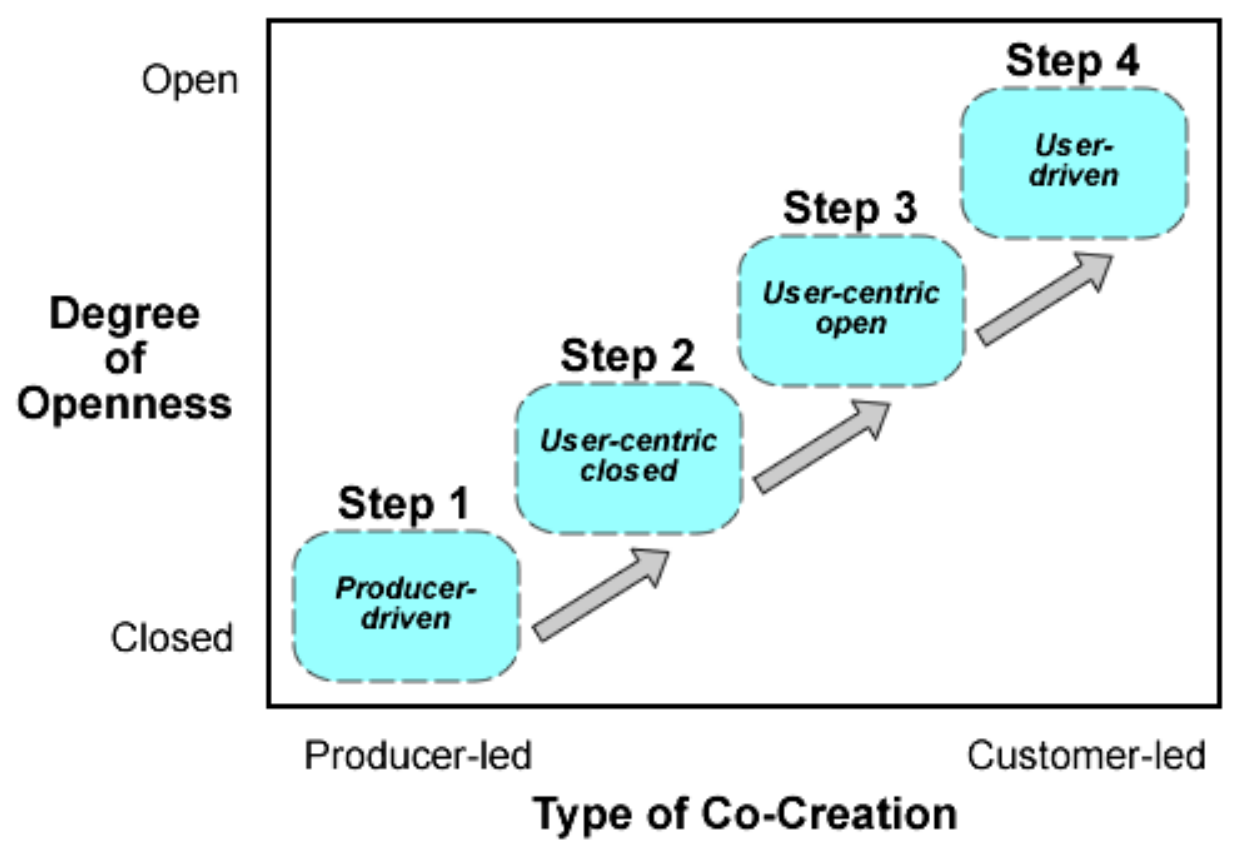

Figure 1. Four steps to becoming an open innovation company

whose role is to purchase and consume the firm's products and services. The contacts in customer firms are not the actual users of the product or service. Users' knowledge and use experiences, as well as potential development ideas, fail to flow into the producer organization due to minimal interaction with customers. Because of the restrictive producer-driven culture, the same may apply even if the company collects feedback by conducting market research, customer surveys, or interviews with the customers, because the firm may not apply this information to its development work. This lack of relevant information in development work is somewhat paradoxical, as even producer-driven innovators recognize the value and benefits of understanding users.

Companies operating in the producer-driven step often use intermediaries such as consultants to obtain customer feedback and development ideas. Because companies' co-creation with users is almost non-existent, intermediaries act as agents between the developer firm and the users. Agents collect users' needs and use experiences, then disseminate them to the producer's R\&D department. Our data suggests that the reason for using agents is their ease of use from the producer's perspective. In addition, companies lack the skills, experience, or resources required to interact with their customers in a way that would benefit the parties involved.
Step 2: User-centric closed. In the second step, development work is still led by the producer and is closed, but the role of users is more visible than in the first step. The producer and its partners collect ideas from users through customer surveys and user studies, which often take place in the company's premises. These studies are quite comprehensive and systematically target specific users. Some users are involved in early stages of the development process, whereas others are included in later stages. Producers use pilot testing for new products and services; pilot users include customers as well as the firm's employees, family members, and employees of the firm's partners.

Some business units within the company have ample resources and experience of user involvement while others have none. $R \& D$ management does not have established general procedures for user involvement, and organizational culture fails to support openness in the innovation process. Therefore, the producer expends a lot of effort protecting its intellectual property rights and maintaining knowledge and information strictly inside the organization. User involvement is not the firm's primary objective and it does not have related organization-wide practices.

Step 3: User-centric open. In the third step, development work is somewhat led by customers, but they are disposable in the sense that a given individual is in- 


\section{Managing the Challenges of Becoming an Open Innovation Company}

\section{Mika Westerlund and Seppo Leminen}

volved in the process only once. This step is a major move towards the open innovation model in terms of increased openness. Companies consider users, who are both the firm's current and potential clientele, as an important source of information. Relevant procedures required for user involvement are widespread within the producer organization and user involvement is among the firm's daily routines. Characteristically, the producer understands the value of its users' knowledge and its previous experiences of value co-creation with customers and users are mainly positive.

Nevertheless, the company only involves users in some phases of the innovation process. It selects them purposely for a certain phase on the basis of its needs; the same users do not participate throughout the product or service innovation lifecycle. The chosen users will be excluded from the subsequent phases after it is accomplished, because they quickly learn how to use the newly-developed service or process. Learning discourages them giving critical feedback and suggestions for further improvements. Therefore, finding more and more new pilot users becomes a challenge for the company.

Step 4: User-driven. In the fourth step, development work is led by customers and is open. In this step, a company enters into intense, long-term collaboration with its users and the majority of the firm's innovation activity is grounded on user involvement. Users' latent needs and motives for collaboration in innovation development rise up and become explicable through their efforts. The firm has well-established procedures for user involvement, and value co-creation with its current or potential customers takes place across the organization.

The company's innovation development practices evolve rapidly. Value co-creation is achieved through continuous trial and error, leading to new products and services, concepts, or operational improvements. The producer often tries new ways of operating and if the new methods do not yield improvements, it tries something else. User-driven development work is truly challenging, because the company entirely opens up its processes and procedures. Organized innovation development activities - which targeted specific users in the previous steps - are now open to any interested parties. Still, operation remains largely unorganized for an undisclosed time; it amends and adapts in time by the interests of the participants.

\section{Managing Increased Openness}

In this section, we describe the management challenges that firms face in opening up their development processes during their transition from conventional, project-based development to open innovation.

Conventional $R \& D$ is grounded in projects that bring about new products and services, beneficial change, or added value. Meredith and Mantel (1995; http://tinyurl .com/5v34qld) point out that a project targets a welldefined set of desired end results and a single project itself is non-recurrent. A project is a temporary endeavor, having a defined beginning and end, and it is undertaken to meet unique goals and objectives. The fundaments of project management are based on attaining preset end results and management reaches these goals by using diverse project management tools, methods, and models (Eskerod and Riis, 2009; http://tinyurl.com/5v9t9kx). Companies can decrease perceived uncertainty by running projects through sequential design phases or subprojects, as in the waterfall model (http://wikipedia.org/wiki/Waterfall_model).

Möller and colleagues (2008; http://tinyurl.com/ 3s95gax) show that innovation co-creation can be producer-driven, customer-driven, or in equilibrium. When employing the open innovation model, user input steers the direction of innovation creation processes heavily (Chesbrough, 2003; http://tinyurl.com/ $455 \mathrm{~m} 3 \mathrm{q} 6)$. Open innovation is based on value co-creation with users and the end result of the development work is unforeseeable beforehand, unlike in conventional development projects. Traditional project management methods, where fundamental assumptions of the management are based on a clear measurable goal of a project (Maylor et al., 2008; http://tinyurl.com/ 3ep6os3), fail to apply in the open innovation model.

Hacievliyagil and Auger (2010; http://tinyurl.com/ 667h9o2) stress the impact of open innovation on the management of $R \& D$. Our data on living labs shows that conventional project-based innovation development and the open innovation model differ in many respects. These differences are highly relevant for the firm's management in its attempt to become an open innovation company. Management needs to pay special attention to these differences in order to stress the right aspects during the transformation. The main differences include: 


\section{Managing the Challenges of Becoming an Open Innovation Company}

\section{Mika Westerlund and Seppo Leminen}

1. Objective. Traditional innovation projects aim at firmly pre-defined goals. Managers can evaluate the success of the project by comparing the realized outcomes with the original project plan. Our data indicates that the open innovation model is different. Living labs target undefined objectives, albeit they introduce loose guidelines to initiate and promote collaboration. The objectives can change many times, as they depend on the interaction and collaboration among participants of the living lab. The results may comprise several different outcomes, which were not targeted in the beginning of the development work. The purpose of collaboration is producing products and services or solutions that have better market fit.

2. Control points. Conventional projects apply preset control points for amendments. Project management control points are usually located at the completion of defined tasks within the overall project plan. Because this plan describes the tasks, it heavily limits and guides the timing of changes in the goals and tasks or even the termination of project. Open innovation allows for changes to be made any time during the co-development work. For example, a living lab has few strictly set control points; it is self-organizing and the goals of innovation development change by the users' activity and involvement.

3. Project manager's role. The project manager's role differs clearly between conventional projects and open innovation. In the conventional model, the project manager manages and controls the resources and organizes schedules according to the project plan. Participants of a living lab cannot be managed as though they are personnel, because users join the innovation co-creation work on a voluntary basis. Their participation is often compelled by hedonic motives instead of economic ones. For example, many users do not expect any monetary rewards because they value the opportunity to participate and learn about the development process. Often, users consider that a token gift or formal recognition of their efforts is sufficient reward. Managers need to learn how to motivate users and other participants in living labs, which is challenging and resource intensive.

4. User's role. A conventional innovation development project deems users as objects of study. They join the project in diverse roles at any time during the product development lifecycle, whether the project is an early trend-identification phase or about to launch. Sometimes, end users test and verify products and services even after the launch. Open innovation is different; users are equal to other participants in living labs, as they are genuine co-creators of value. They participate in various intensive analyses concerning their everyday life, as well as in planning and doing the innovation development work.

5. Resources. Innovation resources in traditional projects include those of the firm and its partners, and companies spend these resources on many activities relating to a project plan. While projects emphasize the capability to utilize extant resources timely and efficiently, a living lab requires new resources and capabilities that are obtained or created by integrating the participants' knowledge. Because the goals change radically over time, co-creation in open innovation may necessitate resources that were not anticipated in the beginning. User involvement is resource intensive and a key managerial challenge is to facilitate user communities to generate sufficient support and resources.

6. Management tools. When managing conventional projects, companies can choose from a large assortment of extant methods and tools, such as the stagegate model (http://wikipedia.org/wiki/Stage-gate_model) or project management software like Microsoft Project, which help managers control and monitor the progress of a project efficiently. Open innovation communities make collective decisions about future directions, and control and coordination is usually self-organized. Therefore, companies running or participating in running living labs need to use diverse facilitative methods, work group tools, and relevant groupware.

\section{Conclusion}

This article investigates co-creation of innovations with users. We examined the challenges firms face when they transform from conventional in-house developers to open innovation companies. A living lab is a real-life test and experimentation environment where users and producers co-create innovations. With data from small and large high-tech firms using the living lab approach, we found four distinctive steps in becoming an open innovator.

We argue that, although a firm can start or stop at any step, the path from closed to open modes of innovation evolves step by step for pragmatic reasons. It spreads out the degree of change in culture and practices, because the transformation is challenging and takes time and effort. Companies must first de-learn their current 


\section{Managing the Challenges of Becoming an Open Innovation Company}

\section{Mika Westerlund and Seppo Leminen}

practices and possibly seek new customer domains that differ from their current market. For small companies, the change towards an open innovator is easier than for large firms, as they are often more agile and less restricted by current markets and practices due to their smallness and newness.

However, the main challenges for any company include establishing a new organizational culture and mindset as well as providing facilities that support increased openness. Traditional project management tools are insufficient for the purpose. Therefore, managers of companies in any industry that intend to become open innovators should apply groupware tools that facilitate and motivate all participants of innovation co-creation. Companies that already use agile development methods probably adapt to open modes of innovation quicker than those relying on plan-driven methods, because they have more adaptive and responsive organizational culture.

\section{About the Authors}

Mika Westerlund, D. Sc. (Econ.), holds positions as Postdoctoral Scholar in the Haas School of Business at the University of California Berkeley and Postdoctoral Researcher in the School of Economics at Aalto University. Mika earned his doctoral degree in Marketing from the Helsinki School of Economics. His doctoral research focused on software firms' business models and his current research interests include open innovation, business strategy, and management models in high-tech and service-intensive industries. Results from his research are reported in numerous scholarly journals.

Seppo Leminen, D. Sc. (Econ.), Lic. Tech., holds positions as Principal Lecturer at the Laurea University of Applied Sciences and Adjunct Professor in the School of Economics at Aalto University. Seppo holds a doctoral degree in Marketing from the Hanken School of Economics and a licentiate degree in Information Technology at the Helsinki University of Technology. His research and consultation interests include value co-creation and capture with users as well as relationships, services, and business models in marketing. He runs various living lab and business model projects in ICT and media industries.

Citation: Westerlund, M. and Leminen, S. 2011. Managing

the Challenges of Becoming an Open Innovation

Company: Experiences from Living Labs. Technology

Innovation Management Review. October 2011: 19-25. 